\title{
Applications of CBR in Oil Well Drilling: A General Overview
}

\author{
Samad Valipour Shokouhi ${ }^{1,3}$, Agnar Aamodt ${ }^{2}$, and Pål Skalle ${ }^{1}$ \\ ${ }^{1}$ Department of Petroleum Technology (IPT) \\ ${ }^{2}$ Department of Computer and Information Science (IDI) \\ Norwegian University of Science and Technology (NTNU) \\ ${ }^{3}$ Verdande Technology AS: Stiklestadveien 1- Trondheim, Norway \\ valipour@ntnu.no, samad@verdandetechnology.com, \\ agnar.aamodt@idi.ntnu.no, pal.skalle@ntnu.no
}

\begin{abstract}
This overview of different applications of CBR in petroleum engineering is based on a survey and comparative evaluation of different successful applications of CBR. The number of papers and research groups is indicative of importance, need, and growth of CBR in different industries. Applicationoriented research in the area of case based reasoning has moved mature research results into practical applications. In this paper we present the evolving story of CBR applied in petroleum engineering especially in drilling engineering. Drilling engineering contains several potential domains of interest, in which CBR can be employed successfully.
\end{abstract}

Keywords: Case-based reasoning, oil well drilling.

\section{Introduction}

Case-based reasoning (CBR) is defined as the branch of artificial intelligence (AI) concerned with solving problems by reuse of past experiences. Case-based reasoning $(\mathrm{CBR})$ is an approach to problem solving and decision making where new problems are solved by finding one or more similar previously solved problems, called cases, and re-using them in the new problem situation [1]. CBR may be used on its own, or integrated with other reasoning modalities to provide more accurate results by compensating the shortcomings of one approach through use of the strengths of another [2].

The aim of the study reported here is to show what possible benefits CBR can provide to the oil and gas drilling industry. The number of publications on the application of CBR in drilling operations indicates that this is a potential method to reduce cost of drilling, and increase safety of the drilling operation, by using previous experiences, hidden in reports and/or known by experts.

Oil and gas are the main energy sources in many countries. To supply world oil consumption, new wells are continuously demanded. Such needs have motivated and inspired people around the world to employ artificial intelligence in drilling 
operations. Oil well drilling is a complex operation. Problems frequently occur when drilling several kilometers through different geological formations. Each well may experience both similar and new problems during the drilling operation. Offshore drilling of an oil well is also an expensive operation, costing typically 250,000 US\$ per day per rig. Access to experts for the purpose of solving problem and knowledge acquisition is limited.

\section{History of CBR from Academia to Industry}

CBR enables utilization of specific knowledge of previously experienced, concrete problem situations. A CBR system requires a good supply of cases in its case database. The retrieval task starts with a problem description, and ends when a best matching previous case has been found. A new problem is solved by finding a similar past case, and reusing it in the new problem situation. Sometimes a modification of the solution is done to adapt the previous solution to the unsolved case. It is important to emphasize that CBR also is an approach to incremental and sustained learning; learning is the last step in a CBR cycle [1], [3]. A CBR system can also enhance its reasoning power through the explicit representation and use of generalized knowledge about a specific domain. A classical example is the CASEY system, a medical application to diagnose heart failures [4]. Later, other frameworks for building knowledgebased systems that integrate CBR with rule-based reasoning (RBR) and model-based reasoning (MBR) were introduced by other groups such as [5] and [6].

The CBR approach was initiated roughly 35 years ago, assuming the work of Schank and Abelson [7] could be considered the origins of CBR. Several academic studies were triggered, including some with ambitions of commercialization of their own applications in the future. One of the early successful and influential applications was at Lockheed, a US aerospace company [8]. Modern aircrafts contain parts made of composite materials which must be cured in large industrial autoclaves. These parts have different characteristics requiring different autoclave heating and cooling profiles. This is complicated by the fact that many parts need to, for economical reasons, be placed together in a single large autoclave, and fact that the parts interact to alter the heating and cooling characteristics of the autoclave. Operators of Lockheed's autoclaves relied upon previous successful parts layouts to inform how to layout the autoclave. They were inspired to develop CLAVIER, the system to assist autoclave operators to reuse previously successful loadings. New successful layouts provided by operators were added to a library to improve performance of CLAVIER. The system retrieved or adapted successful layouts in $90 \%$ of the time. The results indicated that the developed system had capability to solve problems. Note that mistakes were so costly in the domain of application, that CLAVIER was successfully served to other companies[8], [9].

There is a growing trend to employ new approaches in oil well drilling to reduce operational costs by using previous experiences gained from either previously drilled wells or on wells being currently drilled. CBR has been widely applied in the drilling industry with different focuses, described next. However, it is somewhat applied in this industry in the same proven manner as in other domains, e.g. as in aerospace through CLAVIER. 
Applications of CBR in the oil and gas industry are shown on the right side of Fig.1. It further demonstrates the evolution path of the projects that we studied. They vary from methodology to field evaluated phase. The last phase, field evaluated, is synonymous with systems that have been commercialized like CLAVIER.
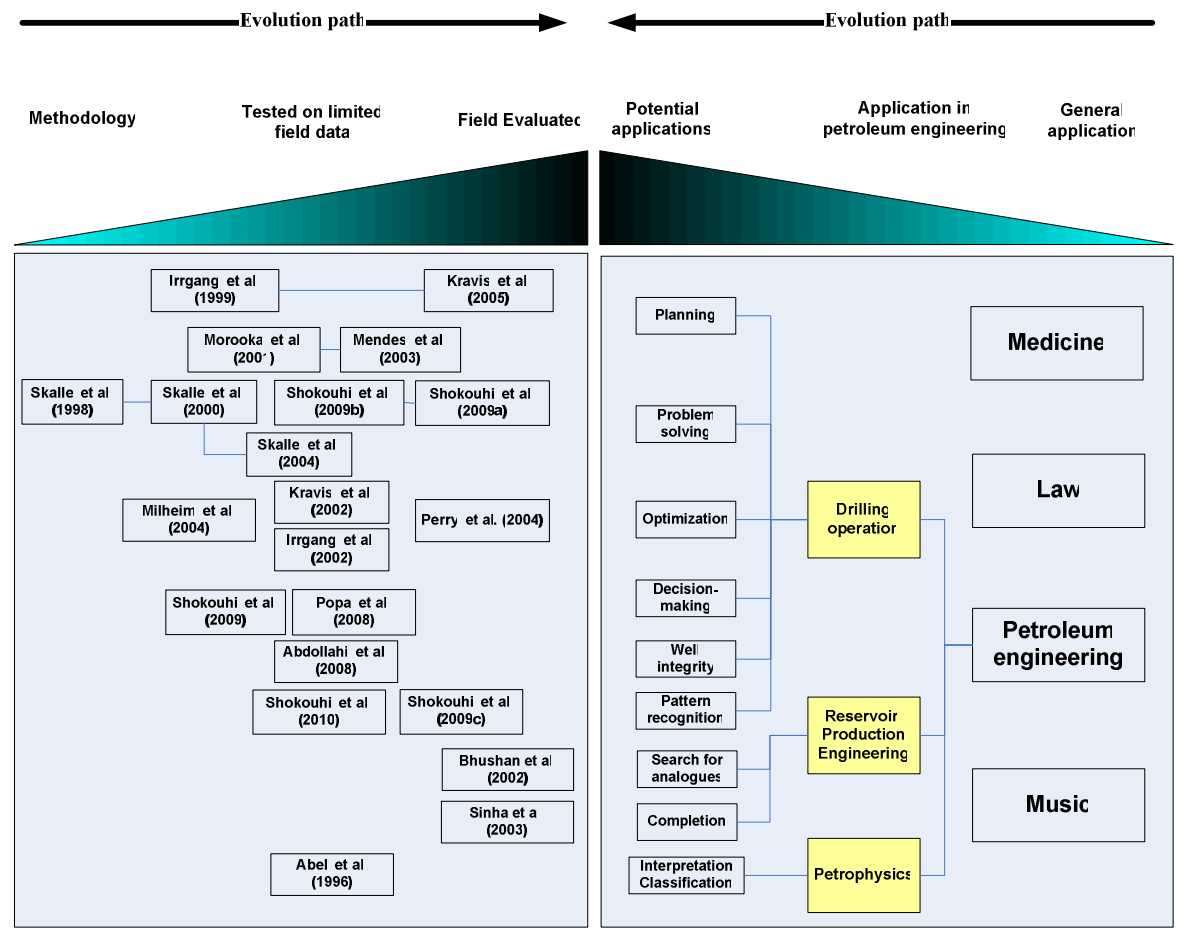

Fig. 1. Summary of CBR used in this study

In section 3 we review applications of CBR in drilling operations. Admittedly, the list may have been somewhat flavoured by the research in our group, but other wellknown work has also been included to the degree their documentation has been easily available. Section 4 explains the applications of CBR in other domains of petroleum engineering. The last section summarizes and concludes on the CBR's state of the art in petroleum engineering.

\section{Applications in Drilling Operation}

A good implementation of CBR will inevitably lead to facilitating solution of repetitive problems. Advanced technologies and equipments are invented and employed in the drilling industry to reduce cost of drilling operations. Drilling an offshore well may typically take one month involving high investments. Generally speaking, the 
drilling industry is a technology dependent industry. Therefore, any sorts of tools or equipments that can improve the drilling operation are essential and are demanded during planning and during plan execution. Case-based reasoning has shown to provide effective support for several tasks related to drilling. Optimization of drilling plans can be achieved through CBR. The CBR method is also used for solving operational problems which require a good description of problematic situations. The information gathered via the problem analysis process may be used in order to make decision. Cases in a CBR system can be kept to predict upcoming situation through sequences.

The potential applications of CBR in drilling operations, mentioned above, were demonstrated by different groups around the world and will be presented in the following sections more in detail.

\subsection{Planning}

Planning a well in an optimal manner is a complex task and highly experienced engineers are needed. As well as using all the information from other disciplines, such as seismic, the experience and analysis of neighboring wells is essential for a good well plan; ' good planning- few problems'".

CBR has been tried in the oil well drilling domain by different groups around the world. One of the first applications of CBR was documented by CSIRO [10] and later refined by [11]. The technique was applied to derive alternate drilling plans based on previously drilled wells. Each well was represented as one case. A case structure has three levels. The three levels are: groups of cases, groups of attributes and groups of defined drilling phases and operations. The proposed system, Genesis, can use multiple cases at varying levels of generalization. Moreover, it uses automated tools to extract knowledge and indexes for the case base from text data.

Mendes et al. presented an application of CBR in offshore well design. The result of that work was a formalization of the methodology for planning of an oil well in a case-based reasoning context. They used fuzzy set theory for the indexing and matching of index features [12].

Mendes and his colleagues implemented a genetic algorithm to determine the proper trajectory and all the pertinent information for drilling. The initial population is the retrieved cases via CBR. It should be noted that the proposed well trajectories have to be designed by other well-known simulators starting from the well created by the genetic algorithm [13]. The current system is a continuation of past work [14], which was moved from research into a real world experimental setting.

\subsection{Operation Problem Solving}

Problems frequently occur when drilling several kilometers through different geological formations. The sensors installed up at the surface and downhole in the drillstring help to run the drilling process smoothly. Data via sensors are transmitted and translated to a viewable format to be interpreted by the drilling crews. In addition to realtime data, documents such as daily drilling reports and end of well reports are reported for every single well. They are valuable resources in which the solutions for 
most of the past problems are expressed. Description of situations is made through both reports, consisting of description of the problems and proposed solution, or through integration of the real-time data and reports. In most CBR approaches, an abnormal situation is considered a place to make a case. It means that whenever a new problem occurs, a case is built and stored in the case database, to be used for the reasoning routine. In addition, some non-problem cases are often also stored. In particular, it is used to store "good" cases that are somewhat similar to "bad" cases, in order to better discriminate between problem and non-problem. In brief, real episodes, previously (re)solved situations, are the core of the reasoning process, in which problematic situations are explained.

Skalle along with his colleagues [15] pioneered the employment of the CBR method in the drilling industry. The work was at the conceptual and design levels. Stuck pipe incidents from an operator for six years were statistically analyzed. Statistical analyses led them to select parameters for cases and build a knowledge model. In this paper there was not any case matching assessment and basically it was a statistical analyses with a focus on development of the knowledge model. Two years later, they implemented the system for prevention of unwanted events in the domain of offshore oil well drilling. They introduced how to make a case in oil well drilling, mostly based on static parameters. Static parameters do not change much between measurements [16]. Their focus was on lost circulation, which means that some of the drilling fluid that always fills the gap between the drillstring and the well wall gets lost into fractures in the geological formation. They built fifty cases on the basis of information from one North Sea operator. A general domain model was used to match non-identical features that were related in the model. The integrated reasoning method is referred to as knowledge-intensive CBR (KiCBR) [6]. The CREEK framework for building knowledge-based systems that integrate CBR with model-based reasoning (MBR) was described more in detail and implemented in the drilling domain by Skalle's group [17].

In 2009, Shokouhi and his colleagues utilized a newly developed version of CREEK to integrate CBR and MBR. In this work static parameters along with dynamic parameters i.e., they can change more often, were used. Hole cleaning episodes were tagged as the problematic situation in this research work. To evaluate the case matching process, cases were categorized and labeled with respect to their downtime. It showed that KiCBR improved the retrieval process and increased the accuracy more than case based reasoning alone. It also presented how to determine the most probable root causes of poor hole cleaning episodes on basis of the knowledge model. They found how integration of MBR and CBR could improve the case matching process [18]. In late 2009, two types of KiCBR were introduced and compared to other reasoning approaches such as plain CBR and plain MBR. The aim was to obtain the best approach of reasoning in terms of the accuracy of the case matching process. The semantic network for the drilling domain was created and all the entities are binary linked. KiCBR tried to expand the set of features in the input cases through the semantic network model. The study showed that the integration of different reasoning methods improved the reasoning better than plain CBR and MBR alone [19]. 


\subsection{Optimization and Execution of Drilling Process}

Optimization of the drilling process is another application of CBR in the drilling industry. A well is being drilled in an efficient way if all the information and knowledge about drilling is utilized. Drilling performance optimization requires all the related knowledge to identify and diagnose barriers to drilling performance.

Milheim and Gaebler implemented an experience transfer tool (heuristic simulation approach) in the oil well drilling domain, based on data sets of 22 actual wells [20]. The accumulated data are treated statistically and fitted to a model based on combining human thought, artificial intelligence and heuristic problem solving. The paper presents the methodology through transformation of 22 sets of well data into a heuristic data set (activated data set). The work had a great potential to be implemented into any geological domain or specific types of drilling process.

Kravis et al. developed software for assessment of overall well quality. By means of a CBR technique, previous, analogous wells or aspects of a well are selected through similarity matching, and adapted to new wells [21]. A comprehensive set of quality measures has been derived and tested on a global database containing wells from all over the world.

Perry et al. [22] describes the development of a case-based knowledge for drilling performance optimization. A system was designed to represent all pertinent information to be used. Project documents, well summary documents, and technical lesson documents are three levels of documents in the knowledgebase hierarchy. The last one, technical lesson documents contain "case" where the lessons were learned from the analysis of the particular drilling application. This knowledge-base system enables clients, e.g. engineers, to work smarter by identifying and implementing proven solutions to the drilling problems at varying phases; planning phase, implementation phase, and post well phase.

\subsection{Decision-Making}

Every decision-making process provides a final option and requires identifying all options beforehand. In this regard, [23] presented a specific application of CBR to determine the optimum cleaning technique for sanded/ seized failures. These failures occur when unconsolidated reservoir sands flow into a well and cause the pump to become stuck. To correct the situation they needed to decide one out of three options; bail, washback or foam. The job length and job costs for each method were significantly different. They presented an application of CBR for planning and execution of well interventions, i.e., production operations, in order to improve the decisionmaking process. In this paper a CBR and a rule-based system are integrated. Rules (IF statements) are used for adaptation of the most common solution proposed by the CBR system. A large database for reasoning assessment was built. Data from almost 5000 well interventions over a period of three years were collected and analyzed. A small subset of historical cases was taken from the database to evaluate the proposed solution with the actual results. According to the similarity assessment, $80 \%$ of the cases were correctly assigned the successful cleaning method. The system presented by Popa was under development and has not been implemented in the field using the revise and retain steps. 
Another research work was launched by [24] that showed the procedure of the case building process and determination of root causes of poor hole cleaning. Three main groups were chosen and a CBR system was used to distinguish between them. However, discrimination among these three groups is a difficult task. They presented some examples of analyses in which the system could enhance the decision-making process by retrieving cases from the correct groups.

\subsection{Well Integrity}

Abdollahi et al. opened a new window for the application of CBR in the petroleum engineering domain. They explained the applicability of CBR for diagnosis of well integrity problems in order to reduce the risk of uncontrolled release of formation fluids into the well through the lifecycle of a well. Well leakages, well control issues and well collisions are named as well integrity. Abdollahi's work focused on well leakages and smartly identified causes of the leakages versus well activities. Predefined rules were used just for determining root causes of the leakage problems. They defined three most phases in which well leakages may occur. The three phases are: installation testing, operations (production / injection), routine testing (in-flow test for BSV and ASV). A causal model is established related to well leakages. Out of 18 cases, 12 solved and 6 unsolved cases were built and used in case matching assessment. All the cases were categorized into five groups according to the main cause of leakage. It was inferred that pre-defined rules could integrate with CBR to obtain causes of well leakages [25].

\subsection{Pattern Recognition}

In most CBR approaches, an abnormal situation is considered to make a case. It means that whenever a new problem occurs, a case is built and stored in the case database, to be used for the reasoning routine. One of the issues for the case building routine is to determine the severity of problems. One criticism made to CBR is the subjectivity in the case definition. The objective and advantage of each case for being stored in the case database is not straightforward task. Moreover, building cases is a time consuming process. To reduce this factor, the methodology of a semi-automatic case building and case discrimination process to make a robust case-based reasoning system was introduced and implemented [26]. All cases regardless of their severity of problems are captured. It means that the case database contains diverse cases from high to low risk. It helps to diminish subjectiveness of case building process. Past cases can be retrieved and evaluated sequentially. As the number of cases increases it is necessary to prioritize which cases should be enter into the case base immediately and which should be stored for later inclusion or discard. Shokouhi et al. [27] presented an intelligent system for prediction through sequences. As most problems during drilling operation are depth dependant, the system keeps all the cases and experiences in each defined depth interval to compose sequences of cases. Each sequence is composed of previous, present and next case. The work demonstrated that minor problems might turn into the high risk problems later on. The prediction was done and the methodology showed its ability through the good results which were obtained. 


\section{Applications in Other Domains of Petroleum Engineering}

Over the last few years CBR has also been applied in other domains of petroleum engineering. The paper closes with a summary of related work in reservoir engineering, production engineering and petrophysics.

\subsection{Applications in Reservoir Engineering}

A standard database search engine returns the results whenever the search criteria meet exactly the matches. A CBR system determines the similarity to search for analogues on basis of matching attributes that are not exactly similar. Reservoirs characteristics are not exactly matched. In 2002, [28] applied CBR to globally search for reservoir analogues as an important step in planning new fields. A knowledge sharing tool was developed, called the Smart Reservoir Prospector (SRP). The results are accessed in a web-based system. It allows users in any Shell operating unit to access the detailed information in milli-seconds. The similarity between reservoirs computes through a set of attributes. Moreover, using reservoir analogues can provide benefits at all stages of the hydrocarbon exploration and production lifecycle, such as benchmarking scope, sharing knowledge, understanding uncertainties, finding peers, making decision, and applying lessons learned.

\subsection{Applications in Petrophysics}

A CBR system coupled with a database system was developed to support the interpretation and classification of new rock samples [5]. To provide petrographic analyses, the system achieves its reasoning power through the set of previous cases combined with some other source of knowledge about a certain domain. Information to build cases was provided through optical and electronic microscope analysis, isotopic and chemical analysis and petrophysics. The system was applied in one type of reservoir rocks i.e., sandstone. An interesting extension of this work would be to interpret other kinds of reservoir rocks.

\subsection{Applications in Well Completion}

A CBR framework was developed in Schlumberger to assess the applicability of seven lift methods for different drilling operations such as land and platform [29]. It works through decoupling the well design into a high-level or conceptual design phase and allowing for interactions between phases. Similar tools were developed for assessment of other completion methods as well.

\section{Summary and Conclusion}

In brief, CBR is a recent methodology compared to other computer science branches. Through this review work, it has been pointed out that the CBR methodology in the oil and gas industry has been employed by several groups all around the world. In this paper we present the evolving story of CBR applied in petroleum engineering, especially in the oil well drilling domain. The focus of this paper is to present and evaluate a number of different research efforts that employ the CBR in an attempt to improve the drilling operations. 
The main point deduced from the above applications is that access to the data and information is a major problem in this business. This general overview may help leaders and manager to be more positive about the CBR technique and give "limitless" access to the data and information. A list of potential topics of case based reasoning is covered for employing in research-oriented or industrial-oriented groups.

The study indicates that the integration of different reasoning methods improves the reasoning and the retrieval process substantially.

\section{Acknowledgment}

The authors would like to express appreciation to people from NTNU and Verdande Technology AS, for their help and cooperation in this work.

\section{References}

1. Aamodt, A., Plaza, E.: Case-Based Reasoning: Fundamental Issues, Methodological Variations, and System Approaches. Artificial Intelligence Communications 7(1), 39-59 (1994)

2. Marling, C., Rissland, E., Aamodt, A.: Integrations with case-based reasoning. Knowledge Engineering Review 20(03), 241-245 (2005)

3. Kolodner, J.: Case-Based Reasoning. Morgan Kaufmann Publishers Inc., San Francisco (1993)

4. Koton, P.: Reasoning about evidence in causal explanations. In: Proceedings of the Seventh National Conference on Artificial Intelligence (AAAI 1988), pp. 256-261. AAAI Press, Menlo Park (1988)

5. Abel, M., Reategui, E.B., Castilho, J.M.V.: Using Case-Based Reasoning In A System That Supports Petrographic Analysis. In: Artificial Intelligence in the Petroleum Industry: Symbolic and Computational Applications II, ch. 7 (1996)

6. Aamodt, A.: Knowledge-Intensive Case-Based Reasoning in CREEK. In: Funk, P., González Calero, P.A. (eds.) ECCBR 2004. LNCS (LNAI), vol. 3155, pp. 1-15. Springer, Heidelberg (2004)

7. Schank, R.C., Abelson, R.P.: Scripts, Plans, Goals and Understanding. Erlbaum, Hillsdale (1977)

8. Mark, W.S.: Case-Based Reasoning for Autoclave Management. In: Proceedings of the Case-Based Reasoning Workshop (1989)

9. Watson, I., Marir, F.: Case-Based Reasoning: A Review. The Knowledge Engineering Review 9(4), 355-381 (1994)

10. Irrgang, R., Damski, C., Kravis, S., Maidla, E., Millheim, K.: A Case-Based System to Cut Drilling Costs. In: SPE 56504, Presented at the SPE Annual Technical Conference and Exhibition Held in Houston, Texas (1999)

11. Kravis, S., Irrgang, R.: A Case Based System for Oil and Gas Well Design with Risk Assessment. Applied Intelligence 23(1), 39-53 (2005)

12. Mendes, J.R.P., Guilherme, I.R., Morooka, C.K.: Case-based system: indexing and retrieval with fuzzy hypercube. In: Joint 9th IFSA World Congress and 20th NAFIPS International Conference, Vancouver, vol. 7 (2001)

13. Mendes, J.R.P., Morooka, C.K., Guilherme, I.R.: Case-based reasoning in offshore well design. Journal of Petroleum Science and Engineering 40, 47-60 (2003) 
14. Morooka, C.K., Guilhermeb, I.R., Mendesa, J.R.P.: Development of intelligent systems for well drilling and petroleum production. Journal of Petroleum Science and Engineering 32(2-4), 191-199 (2001)

15. Skalle, P., Aamodt, A., Sveen, J.: Case-Based Reasoning, a method for gaining experience and giving advise on how to avoid and how to free stuck drill strings. In: Proceedings of IADC Middle East Drilling Conference, Dubai (November 1998)

16. Skalle, P., Sveen, J., Aamodt, A.: Improved Efficiency of Oil Well Drilling through CaseBased Reasoning. In: Mizoguchi, R., Slaney, J.K. (eds.) PRICAI 2000. LNCS, vol. 1886. Springer, Heidelberg (2000)

17. Skalle, P., Aamodt, A.: Knowledge-based Decision Support in Oil Well Drilling. In: Proceedings of the ICIIP, International Conference on Intelligent Information Systems, Beijing, October 21-23 (2004)

18. Shokouhi, S.V., Aamodt, A., Skalle, P., Sørmo, F.: Determining Root Causes of Drilling Problems by Combining Cases and General Knowledge. In: McGinty, L., Wilson, D.C. (eds.) ICCBR 2009. LNCS, vol. 5650, pp. 509-523. Springer, Heidelberg (2009); ISSN-0302-9743

19. Shokouhi, S.V., Aamodt, A., Skalle, P., Sørmo, F.: Comparing Two Types of KnowledgeIntensive CBR for Optimized Oil Well Drilling. In: Proceedings of the 4th Indian International Conference on Artificial Intelligence (IICAI 2009), Tumkur, India, December16-18, pp. 722-737 (2009)

20. Millheim, K.K., Gaebler, T.: Virtual Experience Simulation for Drilling - The Concept. In: 52803-MS, SPE/IADC Drilling Conference, Amsterdam, March 9-11 (1999)

21. Kravis, S., Irrgang, R., Phatak, A., Martins, A., Nakagawa, E.: Drilling Parameter Selection for Well Quality Enhancement in Deepwater Environments. In: SPE 77358-MS, SPE Annual Technical Conference and Exhibition, San Antonio, September 29 - October 2 (2002)

22. Perry, P.B., Curry, D.A., Kerridge, J.D., Lawton, J., Bowden, D., Flett, A.N.: A Case Based Knowledge Repository for Drilling Optimization. In: IADC/SPE Asia Pacific Drilling Technology Conference and Exhibition, Malaysia, September 13-15 (2004)

23. Popa, A., Popa, C., Malamma, M., Hicks, J.: Case-Based Reasoning Approach for Well Failure Diagnostics and Planning. In: SPE 114229-MS, SPE Western Regional and Pacific Section AAPG Joint Meeting, Bakersfield, California, USA, March 29 - April 2 (2008)

24. Shokouhi, S.V., Skalle, P.: Enhancing Decision Making in Critical Drilling Operations. Paper SPE 120290, Prepared for Presentation at the SPE Middle East Oil \& Gas Show and Conference Held in the Bahrain, March 15-18 (2009)

25. Abdollahi, J., Carlsen, I.M., Randhol, P., Tenold, E., Haga, H.B., Jakobsen, T.: A CaseBased Approach to Understand the Complexity of Causal Connections Related to Well Integrity Problems. In: IADC/SPE 111129-MS, Presented at the IADC/SPE Drilling Conference, Orlando, Florida, USA, March 4-6 (2008)

26. Shokouhi, S.V., Aamodt, A., Skalle, P.: A Semi-Automatic Method for Case Acquisition in CBR, A Study in Oil Well Drilling. In: Proceedings of the Tenth IASTED International Conference on Artificial Intelligence and Applications, AIA-2010, Innsbruck, Austria, February 15-17, pp. 263-270. ACTA Press (2010)

27. Shokouhi, S.V., Aamodt, A., Skalle, P., Sørmo, F.: Integration of Real-Time Data and Past Experiences for Reducing Operational Problems. In: IPTC 13969, Proceedings of the International Petroleum Technology Conference held in Doha, Qatar, December 7-9 (2009)

28. Bhushan, V., Hopkinson, S.C.: A Novel Approach to Identify Reservoir Analogues. In: European Petroleum Conference, Aberdeen, United Kingdom, October 29-31 (2002)

29. Sinha, S., Yan, M., Jalali, Y.: A Methodology for Integrated Well Planning. In: SPE 85315-MS, SPE/IADC Middle East Drilling Technology Conference and Exhibition, Abu Dhabi, United Arab Emirates, October 20-22 (2003) 